\title{
Darboux transformation for the NLS equation
}

\author{
Tuncay Aktosun* and Cornelis van der Mee ${ }^{\dagger}$ \\ * Department of Mathematics, University of Texas at Arlington, Arlington, TX 76019-0408, USA \\ ${ }^{\dagger}$ Dipartimento di Matematica e Informatica, Università di Cagliari, Viale Merello 92, 09123
}

Cagliari, Italy

\begin{abstract}
We analyze a certain class of integral equations associated with Marchenko equations and Gel'fand-Levitan equations. Such integral equations arise through a Fourier transformation on various ordinary differential equations involving a spectral parameter. When the integral operator is perturbed by a finite-rank perturbation, we explicitly evaluate the change in the solution in terms of the unperturbed quantities and the finite-rank perturbation. We show that this result provides a fundamental approach to derive Darboux transformations for various systems of ordinary differential operators. We illustrate our theory by providing the explicit Darboux transformation for the Zakharov-Shabat system and show how the potential and wave function change when a simple discrete eigenvalue is added to the spectrum, and thus we also provide a one-parameter family of Darboux transformations for the nonlinear Schrödinger equation.
\end{abstract}

Keywords: Darboux transformation, Marchenko equation, Gel'fand-Levitan equation, ZakharovShabat system, NLS equation, nonlinear Schrödinger equation

PACS: $02.30 . \mathrm{Ik}$

\section{INTRODUCTION}

Consider the spectral problem $\mathscr{L} \psi=\lambda \psi$, where $\mathscr{L}$ is a linear ordinary differential operator acting on some function space and $\lambda$ is the spectral parameter. Let us assume that $\mathscr{L}$ contains one or more "potentials" and that such potentials satisfy certain restrictions, e.g. they may vanish in some sense as the spatial variable goes to infinity in case the spatial domain is either the whole line or the half line. The spectrum of $\mathscr{L}$ consists of all $\lambda$-values for which there exists a nonzero solution $\psi$, which is usually called a wave function. Under appropriate restrictions, the spectrum of $\mathscr{L}$ consists of two parts: The discrete spectrum and the continuous spectrum. A wave function corresponding to a $\lambda$ value in the discrete spectrum is usually known as an eigenfunction of $\mathscr{L}$ and hence $\lambda$ is an eigenvalue. In the language of physics such a wave function is known as a bound state and the corresponding $\lambda$-value is the bound-state energy. A wave function corresponding to a $\lambda$-value in the continuous spectrum is usually known as a generalized eigenfunction of $\mathscr{L}$ and hence $\lambda$ is a generalized eigenvalue. In the language of physics such a wave function is known as a scattering state and the corresponding $\lambda$-value is the scattering-state energy.

Suppose that we are interested in perturbing our operator $\mathscr{L}$ into $\tilde{\mathscr{L}}$, another linear ordinary differential operator acting on the same function space, in such a way that the continuous spectra of $\mathscr{L}$ and $\tilde{\mathscr{L}}$ coincide while their discrete spectra differ from each other by a finite set of discrete eigenvalues. Assume that the operator $\mathscr{L}$ contains a coefficient $u(x, t)$, where $x$ is the independent variable and $t$ is a parameter, and we call that coefficient the potential. Under the transformation $\mathscr{L} \mapsto \tilde{\mathscr{L}}$, let us assume

CP1212, Nonlinear and Modern Mathematical Physics, $1^{\text {st }}$ International Workshop

edited by W. X. Ma, X. B. Hu, and Q. P. Liu

(C) 2010 American Institute of Physics 978-0-7354-0755-8/10/\$30.00 
that the potential is transformed as $u(x, t) \mapsto \tilde{u}(x, t)$. Under the same transformation, let us assume that the wave function changes as $\psi(\lambda, x, t) \mapsto \tilde{\psi}(\lambda, x, t)$. The Darboux transformation $[4-7,10,12]$ at the potential level consists of the determination of $\tilde{u}(x, t)$ in terms of $u(x, t)$ and the wave function $\psi(\lambda, x, t)$, where for $\lambda$-values we use only those discrete eigenvalues appearing in the perturbation. For example, if we only add a bound state at $\lambda=\lambda_{1}$ in order to obtain $\mathscr{L} \mapsto \tilde{\mathscr{L}}$, then the Darboux transformation at the potential level consists of determining $\tilde{u}(x, t)$ in terms of $u(x, t)$ and $\psi\left(\lambda_{1}, x, t\right)$. The Darboux transformation at the wave function level consists of determining $\tilde{\psi}(\lambda, x, t)$ in terms of $\psi(\lambda, x, t)$ and the quantities related to or evaluated at the discrete $\lambda$-eigenvalues appearing in the perturbation.

It is possible that the operator $\mathscr{L}$ contains two or more potential terms. In that case, the Darboux transformation at the potential level consists of determining the perturbations of those potentials in terms of the unperturbed potentials and the quantities related to or evaluated at the $\lambda$-values appearing in the perturbation part of the discrete spectrum.

The order of the differential operator $\mathscr{L}$ determines the number of linearly independent wave functions, and a unique wave function can be specified by imposing an appropriate set of conditions, equal in number to the order of $\mathscr{L}$. It is expected that the Darboux transformation at the wave function level enables us to determine the change in each specific wave function for any $\lambda$-value in the continuous spectrum in terms of the unperturbed quantities at that $\lambda$-value and the discrete $\lambda$-values in the perturbation.

The linear differential operator may be given on the whole line, on a half line, or on a finite $x$-interval. Thus, there is no loss of generality in considering only the cases $x \in(-\infty,+\infty), x \in(-\infty, 0), x \in(0,+\infty)$, and $x \in(0, a)$ for some $a>0$. The spectrum of the linear operator $\mathscr{L}$ certainly depends on the boundary conditions imposed, and in fact those boundary conditions, if any, are usually stated in identifying the differential operator and the function space it acts on.

Associated with each eigenvalue added to the spectrum of a linear operator, we have one or more norming constants, depending on the multiplicity of the added eigenvalue. Thus, for example, when we add a simple eigenvalue to the spectrum, we obtain a one-parameter family of Darboux transformations, where the corresponding norming constant acts as the parameter. In case the multiplicity of the added eigenvalue is greater than one, our family of Darboux transformations contain as many parameters as the multiplicity of that eigenvalue.

Our goal in this paper is to review a recent method [4] showing how to derive in a fundamental way Darboux transformations for a wide class of differential operators. Such differential operators are usually associated with a Gel'fand-Levitan integral equation or a Marchenko integral equation or a system of such integral equations. Recall that the Gel'fand-Levitan method [8,9] and the Marchenko method [8,9] arise in the analysis of inverse problems for differential operators, where the goal is to determine a differential operator in terms of a set of its spectral data. Appropriate sets of spectral data may include scattering coefficients, discrete eigenvalues, bound-state norming constants, or other pieces of information related to the discrete and continuous spectra of the differential operator being determined.

For a review of the literature on Darboux transformations we refer the reader to [4-7,10,12] and the references therein. The details of our method are available in [4]. This review paper of ours is organized as follows. In Section 2 we consider a class of 
aforementioned integral equations and show that their resolvent kernels can explicitly be written in terms of the solutions to such integral equations. In Section 3 we show how the solutions to such integral equations change under finite-rank perturbations of their kernels, and we express that change explicitly in terms of the unperturbed quantities and the finite-rank perturbation. In Section 4 we relate the Darboux transformation at the potential level directly to the change in the solutions to integral equations considered in Section 3, and we also relate the Darboux transformation at the wave function level to the Fourier transform of the change in those solutions. In Section 5 we provide the one-parameter family of Darboux transformations at the potential level and at the wave function level when a simple bound state is added to the spectrum.

\section{AN INTEGRAL EQUATION AND ITS RESOLVENT KERNEL}

Let us consider the integral equation

$$
\alpha+\omega+\alpha \Omega=0,
$$

where $\alpha$ is the unknown function, $\Omega$ is the integral operator acting from the right, and the nonhomogeneous term $\omega$ and the integral kernel coincide. Let us assume that (1) is uniquely solvable in some function space. Using $(I+R)$ to denote the corresponding resolvent operator, where $I$ is the identity operator and

$$
R:=(I+\Omega)^{-1}-I,
$$

we can write the unique solution to (1) as

$$
\alpha=-\omega(I+R) .
$$

Let us use $r(x ; y, z)$ to denote the integral kernel of the operator $R$. In considering the integral equation on the interval $(x,+\infty)$, note that (1) and (2) take the form

$$
\begin{gathered}
\alpha(x, y)+\omega(x, y)+\int_{x}^{\infty} d z \alpha(x, z) \omega(z, y)=0, \quad y>x, \\
\alpha(x, y)=-\omega(x, y)-\int_{x}^{\infty} d z \omega(x, z) r(x ; z, y) .
\end{gathered}
$$

If we consider the interval $(-\infty, x)$, then $(1)$ becomes

$$
\alpha(x, y)+\omega(x, y)+\int_{-\infty}^{x} d z \alpha(x, z) \omega(z, y)=0, \quad y<x .
$$

Similarly, if we consider the integral over $(0, x)$, then $(1)$ becomes

$$
\alpha(x, y)+\omega(x, y)+\int_{0}^{x} d z \alpha(x, z) \omega(z, y)=0, \quad 0<y<x .
$$

In general, we cannot expect to express the kernel of an integral operator in terms of the solution to that integral equation. However, in case the nonhomogeneous term and 
the kernel coincide, we will see that, under appropriate conditions we are able to express the resolvent kernel explicitly in terms of the solution. In particular, we consider (1) when $\Omega$ is $N \times N$-matrix valued and $J$-selfadjoint in the sense that

$$
\Omega=J \Omega^{\dagger} J, \quad \omega(y, z)=J \omega(z, y)^{\dagger} J
$$

where the dagger denotes the matrix adjoint (complex conjugation and matrix transpose) and $J$ is an $N \times N$ selfadjoint involution, i.e.

$$
J=J^{\dagger}=J^{-1} .
$$

For example, $J$ may be assumed to have the form

$$
J=\left[\begin{array}{cc}
I_{j} & 0 \\
0 & -I_{N-j}
\end{array}\right],
$$

for some $0 \leq j \leq N$.

Let us use $\mathscr{H}_{p}^{M \times N}$ to denote the complex Banach space of $M \times N$ matrix-valued measurable functions $F:(x,+\infty) \rightarrow \mathbf{C}^{M \times N}$ such that the matrix norm $\|F(\cdot)\|$ belongs to $L^{p}(x,+\infty)$ for a fixed value of $p$ satisfying $1 \leq p \leq+\infty$.

Even though (1) with the constraint in (6) seems somehow restrictive, it turns out that Gel'fand-Levitan equations associated with a wide variety of ordinary differential operators can be converted into the form of (5) and (6). Similarly, Marchenko equations associated with a wide variety of ordinary differential operators can be converted into the form of (3) and (6) or into the form of (4) and (6). In all such cases, we have the following key result indicating that the resolvent kernel $r(x ; y, z)$ can be explicitly written in terms of $\alpha(x, y)$. For the proof we refer the reader to [4].

Theorem 2.1 Assume that (1) is uniquely solvable in $\mathscr{H}_{2}^{N \times N}$ and that $\Omega$ satisfies (6). Then, the operator $R$ appearing in (2) and the corresponding kernel $r(x ; y, z)$ satisfy

$$
R=J R^{\dagger} J, \quad r(x ; y, z)=J r(x ; z, y)^{\dagger} J .
$$

Furthermore, $r(x ; y, z)$ can be expressed explicitly in terms of the solution $\alpha(x, y)$ to $(1)$. In particular, when (1) has the form of (3) we have

$$
r(x ; y, z)= \begin{cases}\alpha(y, z)+\int_{x}^{y} d s J \alpha(s, y)^{\dagger} J \alpha(s, z), & x<y<z, \\ J \alpha(z, y)^{\dagger} J+\int_{x}^{z} d s J \alpha(s, y)^{\dagger} J \alpha(s, z), & x<z<y,\end{cases}
$$

with similar expressions when (1) has the form of (4) or (5).

\section{FINITE-RANK PERTURBATIONS}

Let us consider (1) when (6) is satisfied, and let us perturb the operator $\Omega$ with a finiterank perturbation so that (1) is transformed into

$$
\tilde{\alpha}+\tilde{\omega}+\tilde{\alpha} \tilde{\Omega}=0,
$$


where, because of the finite-rank perturbation, we have

$$
\tilde{\Omega}=\Omega+F G, \quad \tilde{\omega}(x, y)=\omega(x, y)+f(x) g(y),
$$

with $f$ and $g$ are $N \times j$ and $j \times N$ matrices with entries depending on a single independent variable and belonging to $\mathscr{H}_{1}^{N \times j} \cap \mathscr{H}_{\infty}^{N \times j}$ and $\mathscr{H}_{1}^{j \times N} \cap \mathscr{H}_{\infty}^{j \times N}$, respectively. Note that in general $F$ and $G$ do not commute, and hence in general $f g \neq g f$.

In the case of (3), (8) reads

$$
\tilde{\alpha}(x, y)+\tilde{\omega}(x, y)+\int_{x}^{\infty} d z \tilde{\alpha}(x, z) \tilde{\omega}(z, y)=0, \quad y>x,
$$

with similar explicit expressions in the cases of (4) and (5). We are interested in the following question: Can we express the solution $\tilde{\alpha}$ to (8) explicitly in terms of $\alpha, f$, and $g$ ? It turns out that the answer is yes, and this result is so fundamental that it provides a unified approach to derive Darboux transformations for a wide class of linear ordinary differential operators, as we will see.

Let us introduce the auxiliary quantities $n, q, \tilde{g}$, and $\Gamma$, which are all explicitly constructed in terms of $\alpha, f$, and $g$ appearing in (1) and (9), respectively:

$$
n:=f+\alpha F, \quad q:=g+g J \alpha^{\dagger} J, \quad \tilde{g}:=g(I+R), \quad \Gamma:=I+\tilde{g} F,
$$

where we remind the reader that the resolvent kernel of the operator $R$ is already explicitly available in terms of $\alpha$, as stated in Theorem 2.1. Since $f$ is $N \times j$-matrix valued, $g$ has size $j \times N$, and $\alpha$ has size $N \times N$, it turns out that $n$ has size $N \times j, q$ and $\tilde{g}$ each have sizes $j \times N$, and $\Gamma$ has size $N \times N$. In the case of (3), the quantities in (10) are explicitly written as

$$
\begin{gathered}
n(x):=f(x)+\int_{x}^{\infty} d z \alpha(x, z) f(z), \quad q(x):=g(x)+\int_{x}^{\infty} d z g(z) J \alpha(x, z)^{\dagger} J \\
\tilde{g}(x, y):=g(y)+\int_{x}^{\infty} d z g(z) r(x ; z, y), \quad \Gamma(x):=I+\int_{x}^{\infty} d s q(s) n(s),
\end{gathered}
$$

where $r(x ; y, z)$ is the kernel given in (7). In the cases of (4) and (5), the integrals in (11) and (12) are modified appropriately.

The following theorem, whose proof is available in [4], shows that the solution $\tilde{\alpha}$ to (8) can be explicitly expressed in terms of the solution $\alpha$ to (1) and the finite-rank perturbation terms $f$ and $g$.

Theorem 3.1 Assume that (6) is satisfied and let $\alpha$ and $\tilde{\alpha}$ be the unique solutions to the integral equations (1) and (8), respectively. Then, $\tilde{\alpha}$ can be written explicitly in terms of $\alpha$ and the finite-rank perturbation terms $f$ and $g$ appearing in (9) as

$$
\tilde{\alpha}(x, y)-\alpha(x, y)=-n(x) \Gamma(x)^{-1} \tilde{g}(x, y),
$$

where $n, \tilde{g}$, and $\Gamma$ are the quantities defined in (10). Furthermore, we have

$$
\tilde{\alpha}(x, x)-\alpha(x, x)=-n(x) \Gamma(x)^{-1} q(x),
$$




$$
\operatorname{tr}[\tilde{\alpha}(x, x)-\alpha(x, x)]=\operatorname{tr}\left[\frac{d \Gamma(x)}{d x} \Gamma(x)^{-1}\right]=\frac{1}{\operatorname{det} \Gamma(x)} \frac{d \operatorname{det} \Gamma(x)}{d x},
$$

with tr denoting the matrix trace (the sum of the diagonal entries) and det denoting the matrix determinant.

\section{DARBOUX TRANSFORMATIONS}

The integral equation (1) arises [4] in the study of various scattering and spectral problems. Its solution $\alpha(x, y)$ is related to a particular wave function $\Psi(\lambda, x)$ obtained from certain particular solutions to the spectral problem $\mathscr{L} \psi=\lambda \psi$ via a Fourier transformation in $\lambda$. Similarly, the solution $\tilde{\alpha}(x, y)$ to $(8)$ is related to the particular wave function $\tilde{\Psi}(\lambda, x)$ obtained from certain particular solutions to the spectral problem $\tilde{\mathscr{L}} \tilde{\psi}=\lambda \tilde{\psi}$. Consequently, the inverse Fourier transform of (13) expresses the change $\tilde{\Psi}(\lambda, x)-\Psi(\lambda, x)$ in the wave function under the finite-rank perturbation $\mathscr{L} \mapsto \tilde{\mathscr{L}}$, and that inverse Fourier transform yields the Darboux transformation at the wave function level. Since we have the freedom of forming $\Psi(\lambda, x)$ in many different ways from various particular solutions to $\mathscr{L} \psi=\lambda \psi$, the key formula (13) yields the Darboux transformation for any particular solution to the corresponding spectral problem.

The quantity $\alpha(x, x)$ is closely related to the potential $u(x)$ in the differential operator, and $\tilde{\alpha}(x, x)$ is related to the perturbed potential $\tilde{u}(x)$. Hence, the key formula (14) yields the Darboux transformation at the potential level. In case the potentials $u$ and $\tilde{u}$ are scalar valued, (15) yields the Darboux transformation at the potential level.

In case the potential $u$ appearing in the differential operator $\mathscr{L}$ depends on the additional parameter $t$, then both $\alpha$ and $\Psi$ depend on the parameter $t$ as well, in which case we may write $u(x, t), \alpha(x, y, t), \Psi(\lambda, x, t)$, etc. For simplicity, in this section we omit the argument $t$ because the finite-rank perturbation and the Fourier transform can be considered at a fixed value of $t$, and after the results are obtained one can analyze the dependence on the parameter $t$ by visualizing it as the time variable.

Since $\mathscr{L} \psi=\lambda \psi$ has various particular solutions, depending on the particular solution used, the relevant integral equation given in (1) may be (3), (4), or (5). For example, in the case of (3), where $\alpha(x, y)$ has support for $y>x$, the $N \times N$ wave function $\Psi(\lambda, x)$ is related to $\alpha(x, y)$ as

$$
\Psi(\lambda, x):=e^{-i \lambda J x}+\int_{x}^{\infty} d y \alpha(x, y) e^{-i \lambda J y},
$$

where $J$ is the involution matrix appearing in (6). Using the inverse Fourier transform on (16) we get

$$
\alpha(x, y)=\frac{1}{2 \pi} \int_{-\infty}^{\infty} d \lambda\left[\Psi(\lambda, x)-e^{-i \lambda J x}\right] e^{i \lambda J y} .
$$

Similarly, for $\tilde{\alpha}(x, y)$, we have the associated $N \times N$ matrix-valued wave function $\tilde{\Psi}(\lambda, x)$, where

$$
\tilde{\Psi}(\lambda, x):=e^{-i \lambda J x}+\int_{x}^{\infty} d y \tilde{\alpha}(x, y) e^{-i \lambda J y}
$$




$$
\tilde{\alpha}(x, y)=\frac{1}{2 \pi} \int_{-\infty}^{\infty} d \lambda\left[\tilde{\Psi}(\lambda, x)-e^{-i \lambda J x}\right] e^{i \lambda J y} .
$$

Let us introduce

$$
\gamma(\lambda, x):=\int_{x}^{\infty} d s q(s) \Psi(\lambda, s)
$$

We have [4]

$$
\tilde{\Psi}(\lambda, x)-\Psi(\lambda, x)=-n(x) \Gamma(x)^{-1} \gamma(\lambda, x),
$$

where $n$ and $\Gamma$ are the quantities defined in (11) and (12), respectively. The Darboux transformation at the wave function level readily follows from (17). In the cases of (4) and (5) one can proceed in a similar manner to obtain the corresponding Darboux transformation at the wave function level.

Suppose we add a discrete eigenvalue $\lambda_{j}$ with multiplicity $n_{j}$ to the existing spectrum. Then, associated [3,4] with the eigenvalue $\lambda_{j}$, there are $n_{j}$ parameters $c_{j 0}, \ldots, c_{j\left(n_{j}-1\right)}$, usually known as norming constants. The formula (17) shows how the wave function changes from $\Psi(\lambda, x)$ to $\tilde{\Psi}(\lambda, x)$ and the formula (14) shows how the potential changes from $u(x)$ to $\tilde{u}(x)$. For each discrete eigenvalue $\lambda_{j}$ added to the spectrum, there will be an $n_{j}$-parameter family of potentials $\tilde{u}(x)$, where the norming constants act as the parameters. In case several discrete eigenvalues $\lambda_{1}, \ldots, \lambda_{M}$ are added all at once, it is convenient $[3,4]$ to use a square matrix $A$ whose eigenvalues are related to $\lambda_{j}$ for $j=1, \ldots, M$ in a simple manner; it is also convenient $[3,4]$ to use a matrix $C$ whose entries are related to the norming constants $c_{j s}$ for $j=1, \ldots, M$ and $s=0,1, \ldots, n_{j}-1$.

In the case of (3), the quantities $f(x)$ and $g(x)$ appearing in (9) can usually be represented in the form

$$
f(x)=\left[\begin{array}{cc}
0 & B^{\dagger} e^{-A^{\dagger} x} \\
C e^{-A x} & 0
\end{array}\right], \quad g(y)=\left[\begin{array}{cc}
e^{-A y} B & 0 \\
0 & -e^{-A^{\dagger} y} C^{\dagger}
\end{array}\right],
$$

where $A$ is a constant square matrix with all eigenvalues having positive real parts (the bound-state $\lambda$-values are usually obtained [3] by multiplying the eigenvalues of $A$ by the imaginary unit $i$ ), and $B$ and $C$ are constant matrices of appropriate sizes so that the matrix product $f(x) g(y)$ is well defined and given by

$$
f(x) g(y)=\left[\begin{array}{cc}
0 & -B^{\dagger} e^{-A^{\dagger}(x+y)} C^{\dagger} \\
C e^{-A(x+y)} B & 0
\end{array}\right] .
$$

Note that the finite-rank perturbation is determined by the matrix triplet $(A, B, C)$ in this case. In order to determine the Darboux transformation at the potential level, from (14) we see that we need to determine $n(x), \Gamma(x)$, and $q(x)$ explicitly in terms of the unperturbed wave function $\Psi(\lambda, x)$ and the triplet $(A, B, C)$. To determine the Darboux transformation at the wave function level, from (17) we see that we need to determine $n(x), \Gamma(x)$, and $\gamma(\lambda, x)$ explicitly in terms of the unperturbed wave function $\Psi(\lambda, x)$ and the triplet $(A, B, C)$. Such explicit expressions are derived in [4] and are given by

$$
n(x)=\frac{1}{2 \pi i} \int_{-\infty}^{\infty} d \lambda \Psi(\lambda, x) e^{i \lambda J x} \mathscr{N}(\lambda, x)
$$




$$
\begin{gathered}
q(x)=\frac{1}{2 \pi i} \int_{-\infty}^{\infty} d \lambda \mathscr{Q}(\lambda, x) e^{-i \lambda J x} J \Psi(\lambda, x)^{\dagger} J \\
\Gamma(x)=I-i \int_{-\infty}^{\infty} d \lambda \int_{-\infty}^{\infty} d \mu \int_{x}^{\infty} d y E(\lambda, \mu, y) e^{i \mu J y} \mathscr{N}(\mu, y), \\
\gamma(\lambda, x)=2 \pi \int_{x}^{\infty} d s \int_{-\infty}^{\infty} d \lambda E(\lambda, \lambda, s),
\end{gathered}
$$

where the intermediate quantities $\mathscr{N}, \mathscr{Q}$, and $E$ are defined in terms of the triplet $(A, B, C)$ and the unperturbed wave function $\Psi(\lambda, x)$ as

$$
\begin{gathered}
\mathscr{N}(\lambda, x):=\left[\begin{array}{cc}
0 & -B^{\dagger}\left(\lambda I+i A^{\dagger}\right)^{-1} e^{-A^{\dagger} x} \\
C(\lambda I-i A)^{-1} e^{-A x} & 0
\end{array}\right], \\
\mathscr{Q}(\lambda, x):=\left[\begin{array}{cc}
e^{-A x}(\lambda I-i A)^{-1} B & 0 \\
0 & e^{-A^{\dagger} x}\left(\lambda I+i A^{\dagger}\right)^{-1} C^{\dagger}
\end{array}\right], \\
E(\lambda, \mu, x):=\frac{1}{4 \pi^{2} i} \mathscr{Q}(\lambda, x) e^{-i \lambda J x} J \Psi(\lambda, x)^{\dagger} J \Psi(\mu, x) .
\end{gathered}
$$

\section{DARBOUX TRANSFORMATION FOR THE NLS EQUATION}

Consider the Zakharov-Shabat system [1,2,11,13]

$$
\frac{d \varphi(\lambda, x, t)}{d x}=\left[\begin{array}{cc}
-i \lambda & u(x, t) \\
-u(x, t)^{*} & i \lambda
\end{array}\right] \varphi(\lambda, x, t),
$$

where an asterisk is used to denote complex conjugation and the potential $u$ is integrable in $x$ and contains $t$ as a parameter. In this section we will provide the Darboux transformation for (18) both at the wave function level and at the potential level when we add a simple bound state to the potential $u$ at $\lambda=\lambda_{1}$ with $\operatorname{Im}\left[\lambda_{1}\right]>0$ and the norming constant $c_{1}:=c e^{4 i \lambda_{1}^{2} t}$, where $c$ is a nonzero complex constant. Note that $c_{1}$ depends on $t$ even though we do not indicate that dependence explicitly and that $\operatorname{Im}\left[\lambda_{1}\right]$ denotes the imaginary part of the complex constant $\lambda_{1}$. At the wave function level, we will only provide the Darboux transformation for the Jost solution with the asymptotics $\left[\begin{array}{c}0 \\ e^{i \lambda x}\end{array}\right]$ as $x \rightarrow+\infty$. Let us use $\left[\begin{array}{l}\psi_{1}(\lambda, x, t) \\ \psi_{2}(\lambda, x, t)\end{array}\right]$ to denote that Jost solution. The Darboux transformation for any other particular solution to (18) can be obtained by our method summarized in Section 4 and elaborated in [4], but here we will not provide the Darboux transformation for other particular solutions.

It is known that the potential $u(x, t)$ appearing in (18) satisfies the NLS equation (the nonlinear Schrödinger equation)

$$
i \frac{\partial u}{\partial t}+\frac{\partial^{2} u}{\partial x^{2}}+2|u|^{2} u=0
$$


and hence the Darboux transformation at the potential level for (18) will also constitute a Darboux transformation for (19).

Let us write (18) as $\mathscr{L} \phi=\lambda \phi$, where the linear differential operator $\mathscr{L}$ is given as

$$
\mathscr{L}:=i\left[\begin{array}{cc}
1 & 0 \\
0 & -1
\end{array}\right] \frac{d}{d x}-i\left[\begin{array}{cc}
0 & u(x, t) \\
u(x, t)^{*} & 0
\end{array}\right] .
$$

Thus, we are adding a discrete eigenvalue to the spectrum of $\mathscr{L}$ without changing its continuous spectrum. At the potential level, we are interested in determining the resulting potential $\tilde{u}(x, t)$ in terms of $u(x, t), \lambda_{1}, c_{1}, \psi_{1}\left(\lambda_{1}, x, t\right)$, and $\psi_{2}\left(\lambda_{1}, x, t\right)$. At the wave function level we are interested in determining the resulting quantities $\tilde{\psi}_{1}\left(\lambda_{1}, x, t\right)$ and $\tilde{\psi}_{2}\left(\lambda_{1}, x, t\right)$ in terms of $\lambda_{1}, c_{1}, \psi_{1}(\lambda, x, t)$, and $\psi_{2}(\lambda, x, t)$.

We obtain [4] the following one-parameter family of Darboux transformations:

$$
\begin{gathered}
\tilde{u}(x, t)-u(x, t)=\frac{P_{0}}{\left|P_{7}\right|^{2}+\left|c_{1}\right|^{2} P_{8}^{2}}, \\
{\left[\begin{array}{c}
\tilde{\psi}_{1}(\lambda, x, t) \\
\tilde{\psi}_{2}(\lambda, x, t)
\end{array}\right]-\left[\begin{array}{c}
\psi_{1}(\lambda, x, t) \\
\psi_{2}(\lambda, x, t)
\end{array}\right]=\frac{1}{\left|P_{7}\right|^{2}+\left|c_{1}\right|^{2} P_{8}^{2}}\left[\begin{array}{ll}
P_{1} & P_{2} \\
P_{3} & P_{4}
\end{array}\right]\left[\begin{array}{l}
P_{5} \\
P_{6}
\end{array}\right],}
\end{gathered}
$$

where we have defined

$$
P_{7}:=1+i c_{1}\left[\psi_{1}\left(\lambda_{1}, x, t\right) \dot{\psi}_{2}\left(\lambda_{1}, x, t\right)-\psi_{2}\left(\lambda_{1}, x, t\right) \dot{\psi}_{1}\left(\lambda_{1}, x, t\right)\right],
$$

with an overdot denoting the $\lambda$-derivative and

$$
\begin{gathered}
P_{8}:=\frac{\left|\psi_{1}\left(\lambda_{1}, x, t\right)\right|^{2}+\left|\psi_{2}\left(\lambda_{1}, x, t\right)\right|^{2}}{2 \operatorname{Im}\left[\lambda_{1}\right]}, \\
P_{0}:=2 c_{1}\left[\psi_{1}\left(\lambda_{1}, x, t\right)\right]^{2} P_{7}^{*}-2 c_{1}^{*}\left[\psi_{2}\left(\lambda_{1}, x, t\right)^{*}\right]^{2} P_{7}+4\left|c_{1}\right|^{2} \psi_{1}\left(\lambda_{1}, x, t\right) \psi_{2}\left(\lambda_{1}, x, t\right)^{*} P_{8}, \\
P_{1}:=-\left|c_{1}\right|^{2} \psi_{2}\left(\lambda_{1}, x, t\right)^{*} P_{8}-c_{1} \psi_{1}\left(\lambda_{1}, x, t\right) P_{7}^{*}, \\
P_{2}:=\left|c_{1}\right|^{2} \psi_{1}\left(\lambda_{1}, x, t\right) P_{8}-c_{1}^{*} \psi_{2}\left(\lambda_{1}, x, t\right)^{*} P_{7}, \\
P_{3}:=\left|c_{1}\right|^{2} \psi_{1}\left(\lambda_{1}, x, t\right)^{*} P_{8}-c_{1} \psi_{2}\left(\lambda_{1}, x, t\right) P_{7}^{*}, \\
P_{4}:=\left|c_{1}\right|^{2} \psi_{2}\left(\lambda_{1}, x, t\right) P_{8}+c_{1}^{*} \psi_{1}\left(\lambda_{1}, x, t\right)^{*} P_{7}, \\
P_{6}:=\frac{i}{\lambda-\lambda_{1}}\left[\psi_{1}\left(\lambda_{1}, x, t\right) \psi_{2}(\lambda, x, t)-\psi_{2}\left(\lambda_{1}, x, t\right) \psi_{1}(\lambda, x, t)\right] \\
\lambda-\lambda_{1}^{*}\left[\psi_{1}\left(\lambda_{1}, x, t\right)^{*} \psi_{1}(\lambda, x, t)+\psi_{2}\left(\lambda_{1}, x, t\right)^{*} \psi_{2}(\lambda, x, t)\right] .
\end{gathered}
$$

\section{ACKNOWLEDGMENTS}

The research leading to this article was supported in part by the National Science Foundation under grant DMS-0610494 and by INdAM-GNCS. 


\section{REFERENCES}

1. M. J. Ablowitz and P. A. Clarkson, Solitons, nonlinear evolution equations and inverse scattering, Cambridge Univ. Press, Cambridge, 1991.

2. M. J. Ablowitz and H. Segur, Solitons and the inverse scattering transform, SIAM, Philadelphia, 1981.

3. T. Aktosun, F. Demontis, and C. van der Mee, Exact solutions to the focusing nonlinear Schrödinger equation, Inverse Problems 23, 2171-2195 (2007).

4. T. Aktosun and C. van der Mee, A unified approach to Darboux transformations, Inverse Problems 25, 105003 (2009).

5. M. M. Crum, Associated Sturm-Liouville systems, Quart. J. Math. Oxford Ser. (2) 8, 121-127 (1955).

6. P. Deift and E. Trubowitz, Inverse scattering on the line, Commun. Pure Appl. Math. 32, 121-251 (1979).

7. Chaohao Gu, Hesheng Hu, and Zixiang Zhou, Darboux transformations in integrable systems: theory and their applications, Springer-Verlag, New York, 2005.

8. B. M. Levitan, Inverse Sturm-Liouville problems, VNU Science Press, Utrecht, 1987.

9. V. A. Marchenko, Sturm-Liouville operators and applications, Birkhäuser, Basel, 1986.

10. V. B. Matveev and M. A. Salle, Darboux transformations and solitons, Springer, Berlin, 1991.

11. S. Novikov, S. V. Manakov, L. P. Pitaevskii, and V. E. Zakharov, Theory of solitons, Consultants Bureau, New York, 1984.

12. C. Rogers and W. K. Schief, Bäcklund and Darboux transformations: geometry and modern applications in soliton theory, Cambridge University Press, Cambridge, 2002.

13. V. E. Zakharov and A. B. Shabat, A scheme for integrating the nonlinear equations of mathematical physics by the method of the inverse scattering transform, Funct. Anal. App. 8, 226-235 (1974).

Received November 18, 2009; accepted November 25, 2009 